\title{
Relaciones entre ansiedad-rasgo y ansiedad-estado en competiciones deportivas
}

\author{
Relationship among state and trait anxiety in sport competitions
}

\section{Relaçóes entre ansiedade-traço e ansiedade-estado em competiçóes esportivas}

\author{
Francis Ries, Carolina Castañeda Vázquez, María del Carmen Campos Mesa y Oscar Del Castillo Andrés
}

Departamento de Educación Física y Deporte - Facultad de Ciencias de la Educación

\section{RESUMEN}

En esta investigación se pretende comprobar la teoría de la interacción de Spielberger (1972) en el ámbito de la competición deportiva, analizando las relaciones entre ansiedad-estado y ansiedad-rasgo en la competición, según las dimensiones somáticas y cognitivas de la ansiedad-rasgo y ansiedadestado. Para evaluar ambos constructos se utilizó la versión alemana de los cuestionarios WAI-T y WAI-S, en una muestra de 135 deportistas de Luxemburgo. Los resultados obtenidos seńalan que se pueden confirmar las hipótesis de Spielberger (1972), sobre la interacción entre ansiedad-rasgo y ansiedad-estado, ampliándose su validez en el ámbito de las competiciones deportivas. Asimismo, se comprueba la necesidad de separación multidimensional en las componentes somáticas y cognitivas de la ansiedad para el registro de la ansiedad-rasgo competitiva y la ansiedad-estado competitiva. PALABRAS CLAVE

Ansiedad-estado, ansiedad-rasgo, competiciones deportivas, teoría de ansiedad estado-rasgo.

ABSTRACT

This research aims to test the theory of the interaction of Spielberger (1972) in the field of sports competition, analyzing the relationship between state anxiety and trait anxiety in the competition, according to the somatic and cognitive dimensions of trait anxiety and state anxiety. In order to assess both constructs, the German versions of the WAI-T and WAI-S questionnaires were used in a sample of 135 athletes from Luxembourg. The obtained results confirmed Spielberger's (1972) hypothesis of interaction between trait anxiety and state anxiety, extending its validity in the field of sports competitions. Moreover, the need for multidimensional separation of somatic and cognitive components of anxiety was verified for the assessment of competitive trait and state anxiety.

KEYWORDS

State anxiety, trait anxiety, sport competitions, theory of trait-state anxiety RESUMO

Nessa pesquisa pretende comprovar a teoria da interação de Spielberger (1972) no âmbito da competição esportiva, analisando as relaçôes entre ansiedade -traço e ansiedade- estado na competição, segundo as dimensôes somáticas e cognitivas da ansiedade -traço e ansiedade- estado. Para avaliar ambos padróes foram utilizados a versao alemã dos questionários WAI-T e WAI-S, com uma mostra de 135 esportistas de Luxemburgo. Os resultados obtidos apontam que se podem confirmar a hipóteses de Spielberger (1972), sobre a interação entre ansiedade -traço e ansiedade- estado, ampliando sua validade ao âmbito das competiçôes esportivas.

Mesmo que, comprovamos a necesidade da separaçao multidimensional em algumas competencias somáticas e cognitivas da ansiedade para o registro da ansiedade-rasgo competitiva e a ansiedade-estado competitiva.

PALAVRAS CHAVE

Ansiedade-estado, aniedade-traço, competiçoes esportivas, teoria da ansiedade estado-traço.

\section{Introducción}

Spielberger $(1966,1972,1989)$ considera que para definir la ansiedad de forma adecuada hay que tener en cuenta la diferenciación entre la ansiedad como estado emocional y la ansiedad como rasgo de personalidad, y por ello propuso la Teoría de Ansiedad Estado-Rasgo (Cattell y Scheier, 1961). La ansiedad-estado, según Spielberger (1972), es un "estado emocional" inmediato, modificable en el tiempo, caracterizado por una combinación única de sentimientos de tensión, aprensión y nerviosismo, pensamientos molestos y preocupaciones, junto a cambios fisiológicos. Por su parte, la ansiedad-rasgo hace referencia a las diferencias individuales de ansiedad relativamente estables, siendo éstas una disposición, tendencia o rasgo. Contrariamente a la ansiedad es-

Dirección para correspondencia. Francis Ries. Universidad de Sevilla

C/. Pirotecnia, s/n

41013 Sevilla

fries@us.es tado, la ansiedad-rasgo no se manifiesta directamente en la conducta y debe ser inferida por la frecuencia con la que un individuo experimenta aumentos en su estado de ansiedad. En este sentido, los sujetos con alto grado de ansiedad-rasgo perciben un mayor rango de situaciones como amenazantes y están más predispuestos a sufrir ansiedad-estado de forma más frecuente o con mayor intensidad. Los niveles altos de ansiedad-estado son apreciados como intensamente molestos; por tanto, si una persona no puede evitar el estrés que se los causa, pondrá en marcha las habilidades de afrontamiento necesarias para enfrentarse a la situación amenazante. Si la persona se ve inundada por la ansiedad-estado puede iniciar un proceso defensivo para reducir el estado emocional irritante. Los mecanismos de defensa influyen en el proceso psicofisiológico de tal manera, que se modifica o distorsiona la percepción o valoración de la situación. De esta forma y en la medida en que los mecanismos de defensa tengan éxito, las circunstancias se verán como menos amenazantes y se 
producirá una reducción del estado de ansiedad. Asimismo, y de forma general, los sujetos con altos valores de ansiedadrasgo perciben las situaciones y contextos evaluativos como más amenazantes que aquellos otros que presentan menores niveles de ansiedad-rasgo (Pons, 1994; Wadey y Hanton, 2008; Mellalieu y Hanton, 2009; Mellalieu, Neil, Hanton y Fletcher, 2009).

La interacción entre ambos tipos de ansiedad explica por qué la ansiedad-estado puede variar, tanto entre individuos (la característica de ansiedad puede ser diferente ante la misma situación), como individualmente (la misma persona experimenta ansiedad en una situación, pero no en otra). En cuanto a la ansiedad-rasgo, habría que suponer que individualmente varía poco, y, debido a su influencia, las diferencias de ansiedad-estado entre individuos deberían mantenerse ampliamente estables (consistencia relativa). Finalmente, Hackfort y Spielberger (1989) postulan que, en caso de ansiedad-rasgo elevada, se produce un más rápido aumento de la ansiedad-estado.

Muchos estudios en Psicología del Deporte se centraron en la naturaleza multidimensional de la ansiedad (Schwenkmezger, 1985; Martens, Vealey y Burton, 1990; Endler y Kocovski, 2001; Wadey y Hanton, 2008; Cheng, Hardy y Markland, 2009; Grossbard, Smith, Smoll y Cumming, 2009) y diferenciaron entre ansiedad cognitiva y ansiedad somática. La dimensión cognitiva de la ansiedad hace referencia a la incapacidad y dificultad para mantener la atención y concentración, mientras que la dimensión somática de la ansiedad se refiere a las percepciones de los síntomas corporales causados por la activación del sistema nervioso autónomo, como la aceleración del ritmo cardíaco, sudoración, etc. (Martens et al., 1990).

El estudio y la evaluación de la ansiedad competitiva sigue siendo un tema de actualidad y de gran interés en Psicología del Deporte (Abrahamsen, Roberts y Pensgaard, 2006; Partridge y Wiggins, 2008; Abenza, Alarcón, Leite, Ureña y Piñar, 2009; Cheng et al., 2009; Patel, Omar y Terry, 2010; Ramis, Torregrosa, Viladrich y Cruz, 2010; Neil, Mellalieu, Hanton y Fletcher, 2011; Thomas, Picknell y Hanton, 2011). Recientemente, varios autores españoles (Olmedilla, Andreu, Ortín y Blas, 2009; García-Más, Palou, Smith, Ponseti, Almeida, Lameiras, Jiménez y Leiva, 2011) analizaron en sus respectivos estudios la ansiedad competitiva en futbolistas jóvenes.

La ansiedad ha sido típicamente medida a través de autoinformes aunque no sin haber recibido numerosas críticas (Hackfort y Schwenkmezger, 1989; Smith, Schutz, Smoll y Ptacek, 1995). Sin embargo, éstos siguen siendo la medida más popular para la evaluación de la ansiedad por su fácil aplicación, especialmente en los estudios de campo. El más comúnmente utilizado es la Escala de Ansiedad EstadoRasgo (STAI) de Spielberger (1989), la cual ha mostrado una gran validez de constructo y capacidad para distinguir entre los componentes estado y rasgo. Establecida la validez de constructo del STAI y demostrada su sensibilidad para una gran variedad de situaciones incluido el deporte, se han desarrollado distintas escalas de ansiedad específicas para el campo deportivo como por ejemplo el SCAT (Sport Competition Anxiety Test) o el CSAI-2 (Competitive State Anxiety Inventory), ambos de Martens et al. (1990), y posteriormente revisado (CSAI-2R; Cox, Martens y Russell, 2003), o el SAS (Sport Anxiety Scale) de Smith, Smoll y Schutz (1990) y su versión posterior, el SAS-2 de Smith, Smoll, Cumming y Grossbard (2006). Ramis et al. (2010) diseñaron y validaron una versión adaptada al español del SAS-2 en deportistas de iniciación. En su reseńa, De la Vega (2010) resalta la importancia de estas pruebas que se utilizan en la actualidad en el estudio de la ansiedad en el deporte.

Como eje central para la medida de la multidimensionalidad de la ansiedad se encuentra frecuentemente el análisis de la relación ansiedad y rendimiento (Craft, Magyar, Becker y Feltz, 2003; Woodman y Hardy, 2003). Martens et al. (1990) que informaron de evidentes hallazgos empíricos sobre la Teoría Multidimensional de la Ansiedad (MAT), observaron que la ansiedad tanto cognitiva como somática influyen en el rendimiento de manera diferente, a través de una fuerte relación lineal negativa entre ansiedad-estado cognitiva y rendimiento (a mayor inquietud o preocupación, peor rendimiento), y con menor fuerza, una relación de U-invertida entre ansiedad somática y rendimiento (rendimiento deportivo óptimo en caso de ansiedad somática media; peor rendimiento en caso de ansiedad somática baja y alta) (Martens et al., 1990; Craft et al., 2003; Woodman y Hardy, 2003).

Los supuestos centrales de la Teoría de Ansiedad RasgoEstado de Spielberger (1972), con vistas al ámbito deportivo, también quedaron aquí, en gran medida, sin probar. Sin embargo, se hallaron pruebas de la variabilidad de la ansiedadestado competitiva en los seis días previos a una competición y aumentos significativos en la intensidad de la ansiedad-rasgo (Hanton, Thomas y Maynard, 2004). Sólo en un estudio (Hanton, Mellalieu y Hall, 2002) se verificó explícitamente, si, en general, un mayor estado de ansiedad-rasgo en situación de competición va unido a una mayor ansiedad-estado en situaciones concretas de competiciones deportivas. Se encontraron diferencias significativas acordes con las expectativas, a más ansiedad-estado competitiva, mayor ansiedadrasgo competitiva).

Recientemente Brand, Ehrlenspiel y Graf (2009) presentaron un cuestionario específico para la ansiedad competitiva (WAI), el cual permite tanto la diferenciación de ansiedadestado y ansiedad-rasgo competitiva, como su faceta multidimensional. La escala reducida WAI-T (Brand et al., 2009), mide tanto la ansiedad-rasgo competitiva en sus dimensiones "ansiedad somática", "preocupación (ansiedad cogniti- 
va)", como "problemas de concentración". La escala reducida WAI-S (Ehrlenspiel, Brand y Graf, 2009), mide la ansiedadestado competitiva en sus dimensiones "ansiedad somática", "preocupación (ansiedad cognitiva)” y "confianza” (como dimensión negativa de la ansiedad).

En esta investigación pretendemos comprobar la teoría de la interacción de Spielberger en el ámbito de la competición deportiva, analizando las relaciones entre ansiedad-estado y ansiedad-rasgo en la competición y, de modo separado, según las dimensiones somáticas y cognitivas de la ansiedad-rasgo y ansiedad-estado. Para ello se comprobará la estabilidad de la ansiedad-rasgo y la variabilidad de la ansiedad-estado, estudiando la fuerza predictiva de la ansiedad-rasgo para la ansiedad-estado, y analizando la influencia de la ansiedad-rasgo competitiva sobre el aumento de la ansiedad-estado competitiva.

De manera concreta pretendemos comprobar si la ansiedadestado competitiva es más elevada el día de la prueba que unos días antes, es decir, que varía, mientras que la ansiedad-rasgo competitiva permanece estable. Suponemos que la variabilidad y la estabilidad se reflejarán también en el sentido de que las puntuaciones individuales de ansiedad-estado variarán más que las puntuaciones de ansiedad-rasgo. Por último, esperamos que la ansiedad-rasgo prediga la ansiedad-estado, tanto algunos días antes de la competición como cerca de la misma (el mismo día), y se comprobará la predicción de que la ansiedadestado competitiva se incrementa en los días previos a la competición cuanto más acusada sea la ansiedad-rasgo competitiva.

\section{Método}

\section{Participantes}

En este estudio participaron 135 deportistas de ambos sexos, 80 varones y 55 mujeres con edades comprendidas entre 18 y 26 ańos $(M=21.15 ; D . T .=2.61)$ elegidos al azar de la lista de las federaciones deportivas del "Comité Olympique et Sportif Luxembourgeois" (COSL). El 79\% de los deportistas participaba regularmente en competiciones y el $75 \%$ dijo practicar su deporte más de dos veces por semana con una media de 7.23 horas semanales $(D . T .=3.14)$. El 75.6\% indicó que su nivel de participación en competiciones era internacional y nacional. Los deportistas provenían de diversos tipos de deporte: ciclismo (27.3\%), fútbol (20.02\%), baloncesto (14.5\%), gimnasia deportiva (12.4\%), natación (11.9\%), esquí (8.32\%), y judo (5.56\%). Se contactó con los participantes bien de manera directa, bien a través de sus entrenadores, completando aquéllos los cuestionarios de manera voluntaria.

\section{Instrumentos}

Para evaluar la ansiedad-rasgo competitiva de los deportistas luxemburgueses, se utilizó la versión alemana del WAI-T
(Brand et al., 2009) debido a que el alemán es una de las tres lenguas oficiales con mayor representación en Luxemburgo. El WAI-T está compuesto por 14 ítems que abarcan las dimensiones somáticas y cognitivas de la ansiedad-rasgo competitiva y la desconcentración, aunque esta última dimensión no se tuvo en cuenta ya que no formaba parte de los objetivos del presente estudio. Los participantes firmaron un consentimiento informado y se les explicó que este cuestionario mide sus vivencias y pensamientos respecto a las situaciones de competición en general, es decir, cómo se sienten habitualmente antes de una prueba, respectivamente, en qué piensan. Finalmente, las dos subescalas utilizadas alcanzaron en el caso de los deportistas luxemburgueses una consistencia interna de $\alpha=.78$ y de $\alpha=.79$, respectivamente. Para medir la ansiedad-estado competitiva se utilizó el cuestionario WAI-S (Ehrlenspiel et al., 2009), que consta de 12 ítems que miden las dimensiones somáticas y cognitivas, así como la auto-confianza (la cual tampoco entró en el análisis de los datos por no coincidir con los objetivos planteados). Los deportistas firmaron de nuevo un consentimiento informado y se les explicó que este cuestionario registra las vivencias y pensamientos actuales referidos a la próxima competición. La consistencia interna de ambas subescalas utilizadas del WAI$S$ alcanzó también valores aceptables, tanto para la ansiedad somática competitiva $(\alpha=.81)$, como para su dimensión cognitiva $(\alpha=.78)$.

\section{Procedimiento}

Siguiendo las instrucciones de Ehrlenspiel et al. (2009), Brand et al. (2009), y de Martens et al. (1990), los deportistas seleccionados rellenaron ambos cuestionarios (WAI-T y WAI-S) en dos ocasiones, cuatro días antes de la competición y el mismo día de la prueba. La primera recogida de datos se fijó para cuatro días antes de la prueba, de manera que la fecha quedara suficientemente distante de la próxima competición, sin estar por ello todavía bajo la influencia de la prueba anterior. Los participantes fueron cuidadosamente instruidos para que rellenaran el cuestionario siguiendo esas directrices, de forma individual en su casa, inmediatamente después de levantarse. Con ello debía garantizarse que el registro no pudiera resultar viciado por elementos estresantes no relacionados con la competición. Para evitar posibles efectos de orden de preguntas, se cambió el orden de las mismas y de las dos subescalas de forma aleatoria en las dos diferentes recogidas de datos.

\section{Análisis de los datos}

A diferencia del único estudio conocido por nosotros, en el que al menos la predicción arriba citada sobre ansiedad-rasgo y ansiedad-estado también fue investigada referida a la competi- 
ción y con carácter multidimensional (Hanton et al., 2002), en el presente estudio utilizamos path-analysis más exigentes, respectivamente, más concluyentes, para el análisis estadístico de datos, que conllevan la particular ventaja de la consideración simultánea multivariada de la ansiedad-rasgo y ansiedad-estado registrados, y que permiten la representación y descripción de relaciones de efectos tanto parciales como de conjunto

Para comparar la estabilidad intra-individual de la ansiedad-rasgo competitiva y la variabilidad intra-individual de la ansiedad-estado competitiva, se realizan análisis de varianza independientes $2 \times 2$ con medición repetida para las dimensiones somática y cognitiva (Ansiedad-rasgo/ansiedad-estado $\mathrm{x}$ fecha de la toma de datos: 4 días antes de la competición/día de la competición), calculando los valores de las escalas como variables dependientes. Se calcula de nuevo independientemente para ambas dimensiones de la ansiedad si el valor de ansiedad-estado registrado el día de la competición puede deberse al nivel de ansiedad-rasgo medido 4 días antes.

La confirmación de las hipótesis de partida, la comprobación de los modelos de ecuaciones estructurales y el cálculo de los índices de ajuste se realizaron mediante LISREL 8.71. (Jöreskog y Sörbom, 2004). Los parámetros se lograron a través del método de Máxima Verosimilitud. Este método de estimación es uno de los más manejados en el estudio de modelos estructurales puesto que ofrece estimadores consistentes, no sesgados, eficientes e invariantes ante los cambios de escala (Bollen y Curran, 2006). Aunque la aplicación de esta función requiere el cumplimiento de normalidad multivariante en los datos, se ha demostrado que es un método robusto que muestra buenos resultados en las situaciones menos favorables, como es el caso de excesiva kurtosis y bajo tamaño muestral (Hoyle, 1995). No obstante, presenta sensibilidad al tamaño de la muestra, habiéndose constatado que el tamaño ideal para su aplicación se encuentra entre 100 y 200 sujetos (Lévy-Mangin, 2006); por otro lado, el número de sujetos por variable observada que se aconseja en la literatura varía entre 5 y 10 (Bentler, 1989), por lo que en este estudio optamos por elegir una muestra compuesta por encima de 135 sujetos de acuerdo a los indicadores contemplados. Teniendo en cuenta estas consideraciones, la bondad de ajuste se evaluó con los índices de ajuste más aconsejados en la literatura específica (Hu y Bentler, 1999; Beauducel y Wittman, 2005). Para su valoración se utilizaron los siguientes índices: el Root Mean Squared Error of Approximation (RMSEA $\leq .08$ para $N<250$ ), el Standardized Root Mean Square Residual (SRMR $\leq .11$ ), y el Comparative Fit Index (CFI > .95). Para comprobar además la influencia de las dimensiones de la ansiedad-rasgo sobre el aumento de la ansiedad-estado se calculan modelos de regresión lineal con los aumentos en ansiedad-estado como criterio (diferencia de los valores registrados).

\section{Resultados}

Con relación al primer objetivo planteado, acerca de comprobar la estabilidad de la ansiedad-rasgo competitiva y la variabilidad de la ansiedad-estado competitiva, podemos observar, en la figura 1, que tanto la dimensión somática como la dimensión cognitiva de la ansiedad-rasgo competitiva permanecen estables de forma intra-individual, mientras que la ansiedad-estado competitiva varía.

Figura 1: Variabilidad de la ansiedad-rasgo competitiva y ansiedad-estado competitiva

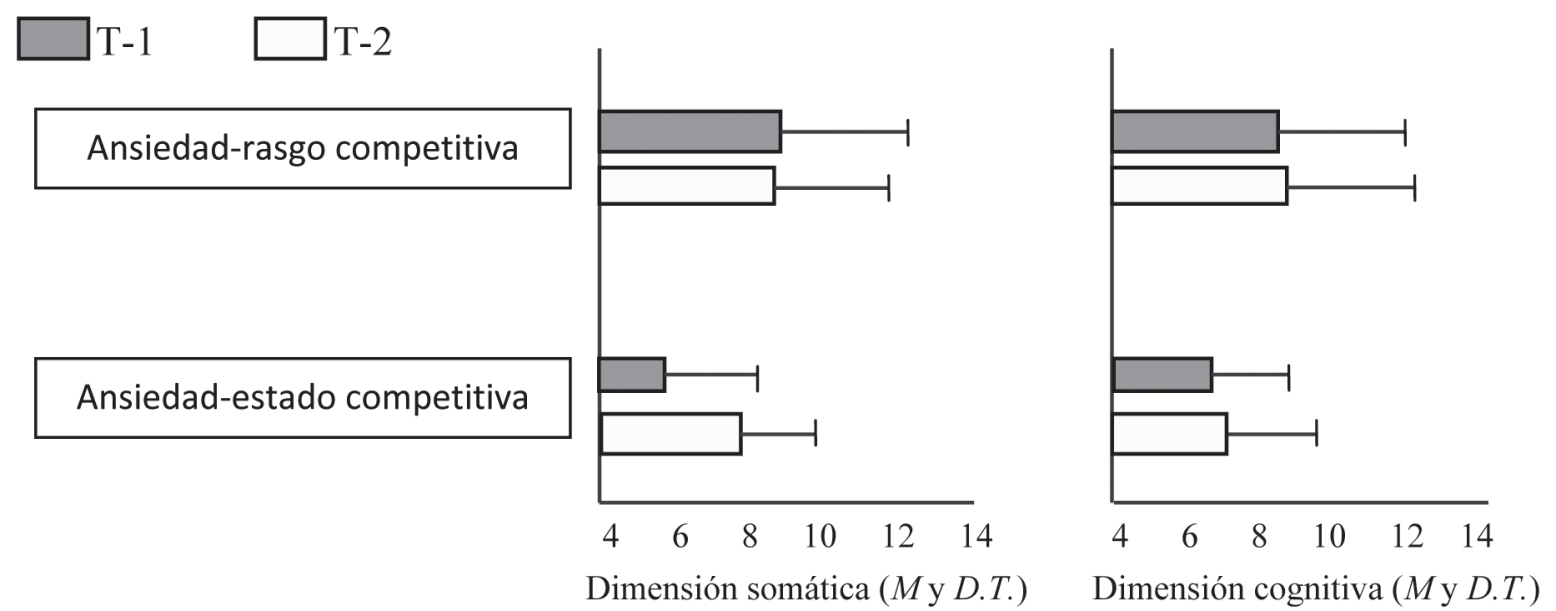

Nota: $(\mathrm{T}-1)$ = recogida de datos 4 días antes de la competición; $(\mathrm{T}-2)$ = recogida de datos el mismo día de la competición; se han representado los valores medios ( $M)$ y la desviación típica (D.T.). 
Encontramos interacciones significativas entre ansiedadrasgo/ansiedad-estado y la fecha de la recogida de datos, tanto en la dimensión somática $F=33.53, p<.01, c^{2}=.17$, como en la dimensión cognitiva $F=7.57, p<.01, c^{2}=.06$. En la figura 2 pueden verse datos acerca de la estabilidad de diferencias entre individuos, y más exactamente, los coeficientes de regresión entre los momentos de registro de la ansiedadrasgo, respectivamente ansiedad-estado. La estabilidad de la ansiedad-rasgo es mayor en su dimensión somática $(r=.57)$ y en su dimensión cognitiva $(r=.59)$, que la de la ansiedadestado en su dimensión somática $(r=.21)$ y en su dimensión cognitiva $(r=.23)$.

Figura 2: Modelo estructural

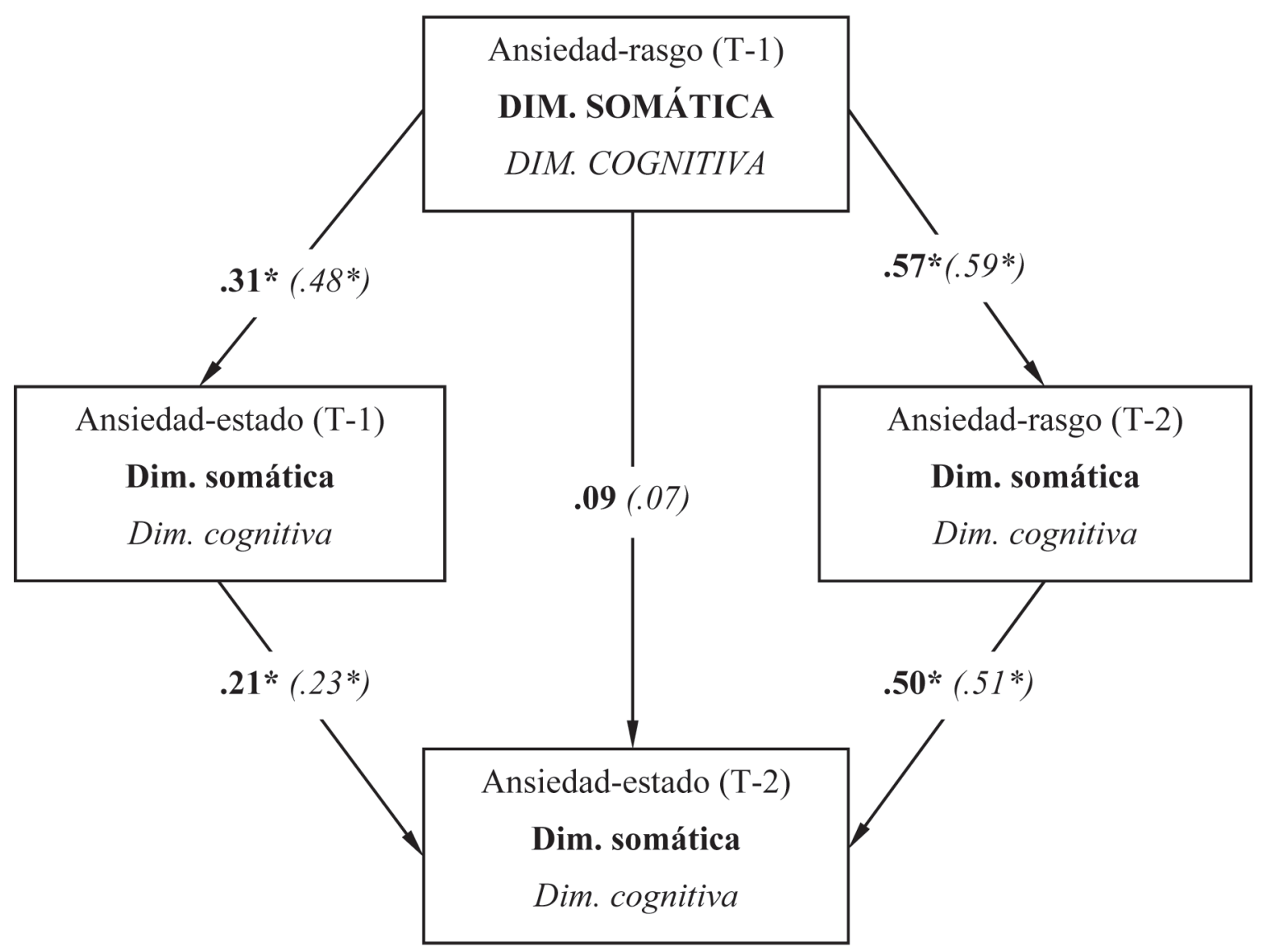

Nota: $($ T-1) = recogida de datos 4 días antes de la competición; $($ T-2) = recogida de datos el mismo día de la competición; DIM. = dimensión; los coeficientes de regresión en negrita corresponden a la dimensión somática de la ansiedad; los coeficientes de regresión en cursiva corresponden a la dimensión preocupación; * $p<.05$

Encontramos un buen ajuste para el modelo teórico de influencia de la ansiedad-rasgo somática sobre la ansiedad-estado somática (tabla 1). Sin embargo, este efecto directo de la ansiedad-rasgo (cuatro días antes de la competición) sobre la ansiedad-estado (día de la competición) es limitado, con un coeficiente de regresión de $r=.09$ y no-significativo $(p>$ $.05)$. Pero si consideramos también los efectos indirectos, podemos calcular un efecto total de $r=.57$ de la ansiedad-rasgo competitiva (cuatro días antes de la competición) sobre la ansiedad-estado (día de la competición). No constatamos un buen ajuste del modelo para la dimensión cognitiva y hemos de rechazarlo en base a los valores de $\chi 2$ y de RMSEA (aunque el índice CFI permitiría aún una suposición). También en este modelo el efecto directo de la ansiedad-rasgo (cuatro días antes de la competición) sobre la ansiedad-estado (día de la prueba) es escaso, con un coeficiente de regresión incluso inferior $(r=.07 ; p>.05)$. Considerando los efectos indirectos, también aquí podemos calcular un efecto total con una correlación de $r=.52$. 
Tabla 1. Índices de bondad de ajuste.

\begin{tabular}{lccccc}
\hline Modelo & $\chi 2$ & $d f p$ & RMSEA & CFI & $S R M R$ \\
\hline Dimensión cognitiva & 11.12 & 1 & $<.01 .17$ & .98 & .058 \\
\hline Dimensión somática & 0.63 & $1.32<.01$ & & .99 & .021 \\
\hline
\end{tabular}

Con una varianza explicada del $10 \%$ y un peso de $\beta=.31$, el predictor ansiedad-rasgo somática en el primer momento de toma de datos (T-1) tiene un efecto significativo $(p<.01)$ sobre el aumento de la ansiedad-estado somática competitiva. Sin embargo, el predictor ansiedad cognitiva, en la primera toma de datos, tiene, con un menor peso $(\beta=.14 ; p<.05)$, un efecto sobre el aumento de la ansiedad-estado cognitiva. En total, el modelo sólo explica el 4\% de la varianza.

\section{Discusión}

Respecto al primer objetivo específico, con el que se pretendía comprobar la estabilidad de la ansiedad-rasgo y la variabilidad de la ansiedad-estado competitiva, de manera que ésta sería más elevada el día de la prueba que unos días antes, se ha comprobado un importante cambio en la ansiedad-estado competitiva cuatro días antes de la competición, y en el mismo día. Acorde con lo esperado, los deportistas de ambos sexos reflejan una mayor ansiedad-estado competitiva en el día de la prueba. También acorde con la hipótesis planteada, las puntuaciones de ansiedad-rasgo competitiva permanecen estables en las dos recogidas de datos.

Estos resultados se confirman también a través del pathanalysis. Los coeficientes de regresión relativamente altos entre las dos recogidas de datos de la ansiedad-rasgo competitiva indican estabilidad en esta característica; coeficientes relativamente bajos entre las dos tomas de datos para la ansiedad-estado competitiva señalan su mayor variabilidad.

En cuanto al segundo objetivo específico de la investigación, los resultados confirman igualmente que la ansiedadrasgo predice la ansiedad-estado. Sin embargo, el efecto directo de ansiedad-rasgo cuatro días antes de la competición sobre la ansiedad-estado en el día de la competición es nosignificativo, mientras que los coeficientes de regresión entre ansiedad-rasgo y ansiedad-estado en el día de la competición son más altos el día de la competición que cuatro días antes de la misma (sobre todo, para la dimensión somática). La ansiedad-rasgo competitiva cuatro días antes de la competición no aporta ningún aumento de la varianza frente a la medida más cercana a la competición. Esto muestra que la predicción de la ansiedad-estado mediante la ansiedad-rasgo medida cercana a la competición, es ampliamente independiente del momento del registro de la ansiedad-rasgo. La predicción, algo mejor en el mismo día de la competición, recomienda mantener una diferenciación conceptual entre ansiedadestado y ansiedad-rasgo porque si la ansiedad-estado ha de ser realmente dependiente de la situación y fluctuante, la correlación con la ansiedad-rasgo no puede permanecer estable (Schwenkmezger, 1985).

Asimismo, se confirman también las predicciones de Spielberger (1972) y la segunda hipótesis de la presente investigación, pues la ansiedad-estado competitiva aumenta más intensamente en personas acusadamente ansiosas pudiendo reproducir más marcadamente esta relación en la dimensión somática de la ansiedad. Sin embargo, los efectos totales deben ser considerados como bajos. También pudo probarse la mayor estabilidad intra-individual de la ansiedad-rasgo competitiva en comparación con el constructo de ansiedad-estado competitiva. Tanto la dimensión cognitiva como la somática de la ansiedad-rasgo competitiva, permanecen invariables, mientras que la ansiedad-estado competitiva aumenta en el transcurso de la semana anterior a la competición. Tal como supusieron Martens et al. (1990), esta modificación de la dimensión somática de la ansiedad-estado competitiva resulta mayor que la de la dimensión cognitiva.

Por tanto, se puede confirmar la suposición de Spielberger (1972), en el sentido de que el aumento de la ansiedad-estado depende de la ansiedad-rasgo. Esta relación se refleja con mayor precisión en la dimensión somática que en la dimensión de preocupación. En los estudios de campo realizados por Schwenkmezger (1985), no se encontró ninguna prueba para ello, de modo que puede deducirse que la influencia teórica esperada de la ansiedad-rasgo sobre el aumento de la ansiedad-estado se basaría en situaciones ambivalentes (aquellas que no permiten una evaluación precisa de amenaza). Nuestros resultados señalan que las competiciones también ofrecen condiciones ambivalentes, por lo menos cuando el valor de amenaza de una competición aún no ha sido completamente evaluado en la pre-competición. La todavía inconclusa valoración de lo amenazante, conduce entonces a una mayor influencia de la ansiedad-rasgo sobre la vivencia actual también en competiciones. Cuanto más acusada sea la ansiedad-rasgo competitiva, más pronto se percibirá la inminente situación como amenazante y más aumentará el estado de ansiedad ante la misma. Posiblemente, el enfoque metodológico elegido contribuyó a poder observar lo significativo de la ansiedad-estado en individuos con alta ansiedad-rasgo: el hecho de rellenar los cuestionarios en el propio hogar (nada más levantarse por la mañana, debido a la distancia del centro deportivo) puede haber dejado más espacio para una evaluación ambivalente de la amenaza situacional. Por otra parte, el recurso a técnicas de análisis de regresión, respectivamente, 
la renuncia a dictomización de la ansiedad-rasgo competitiva, seguramente contribuyó a una mayor potencia de prueba (MacCallum, Zhang, Preacher, y Rucker, 2002), mejorando así las posibilidades de descubrir los efectos menores.

La investigación realizada presenta algunas limitaciones. Su diseño es de naturaleza no -experimental debido a que los datos han sido obtenidos mediante autoinformes. En futuros estudios resulta necesario el planteamiento de metodologías complementarias que permitan analizar la validez predictiva de las medidas obtenidas en ansiedad-rasgo y ansiedadestado mediante autoinforme. Del mismo modo, deben estudiarse limitaciones a la interpretación de los resultados que pudieran derivarse de ciertos aspectos de la ejecución: no podemos partir de la base de que todos los individuos cumplieran los protocolos de trabajo según lo previsto, y por lo tanto desconocemos cuándo y en qué entorno procedieron los atletas realmente a rellenar los cuestionarios. Otra imprecisión podría haberse producido a través del cumplimiento de los cuestionarios uno a continuación del otro, porque podrían haberse favorecido efectos de transferencia que originaran la sobrevaloración de la concordancia de ambas características de ansiedad. Se intentó mantener este efecto tan bajo como fuese posible mediante cambios en la sucesión de los cuestionarios realizados al azar. Por esta razón podría más bien haberse minusvalorado algo la relación, pues es sabido que la estabilidad de la ansiedad-rasgo se reduce con el tiempo (Brand et al., 2009).

En conclusión, el presente estudio confirma las hipótesis de Spielberger (1972), sobre la interacción entre ansiedadrasgo y ansiedad-estado, y amplía su validez en el ámbito de las competiciones deportivas. Los estudios también justifican que, para el registro de la ansiedad-rasgo competitiva y de la ansiedad-estado competitiva, es necesaria la separación multidimensional en las componentes somáticas y cognitivas de la ansiedad. Finalmente, el estudio tiene su trascendencia práctica, dando apoyo al diagnóstico comprehensivo de la ansiedad competitiva. Para comprender y asimilar el fenómeno de la ansiedad competitiva de los deportistas, parece necesario abarcar sus diferentes aspectos (que deben ser integrados y transferidos a las decisiones de intervención).

Dado que, aparentemente, las competiciones pueden originar diferentes aumentos en la ansiedad-estado, sería interesante examinar además otros potenciales factores de influencia, como es el caso específico de las mujeres, que suelen tener unos niveles más elevados de ansiedad-estado y ansiedad-rasgo, por lo que existen normas diferenciadas. La posible influencia del evento o la competición, en función de las características o el nivel de la misma (Sarriugarte, 1999; Guillén, Santana y Bara, 2006), la influencia del nivel del rival o del equipo rival (Abenza, Alarcón, Leite, Ureña y Piñar, 2009), o, por otro lado, la edad, y con ella una mayor experiencia competitiva, podrían reducir la ambivalencia de las competiciones también en la pre-competición (Guillén, Sánchez y Márquez, 2003; Guillén y Sánchez, 2009).

A partir de una ansiedad-rasgo competitiva relativamente estable, se podrían predecir diferencias en la vivencia y desarrollo de la ansiedad-estado, y, con ayuda del cuestionario WAI-T (Brand et al., 2009), se podría diagnosticar si deportistas de ambos sexos tienden a reaccionar con ansiedadestado competitiva, pudiendo de este modo identificar a los deportistas "expuestos al riesgo", y, también, implementar planteamientos básicos de intervención.

\section{Referencias}

1. Abenza, L., Alarcón, F., Leite, N., Ureña, N. y Piñar, M. (2009). Relación entre la ansiedad y la eficacia de un equipo de baloncesto durante la competición. Cuadernos de Psicología del Deporte, 9 (suppl.), 51.

2. Abrahamsen, F.E., Roberts, G.C. y Pensgaard, A.M. (2006). Achievement goals and gender effects on multidimensional anxiety in national elite sport. Psychology of Sport and Exercise, 9, 449-464.

3. Beauducel, A. y Wittmann, W.W. (2005). Simulation study on fit indexes in CFA based on data with slightly distor- ted simple structure. Structural Equation Modeling, 12, 41-75.

4. Bentler, P.M. (1989). EQS: Structural Equations Program Manual. Los Angeles: BMDP Statistical Software.

5. Bollen, K.A. y Curran, P.J. (2006). Latent curve models: A structural equation perspective. New York: Wiley.

6. Brand, R., Ehrlenspiel, F. y Graf, K. (2009). Das Wettkampf-Angst-Inventar. Manual. Bonn: BISp.

7. Cattell, R.B. y Scheier, I.H. (1961). The meaning and measurement of neuroticism and anxiety. New York, NY: Ronald Press.

8. Cheng, W.K., Hardy, L. y Markland, D. (2009). Toward a three - dimensional conceptualization of performance anxiety: Rationale and initial measurement development. Psychology of Sport and Exercise, 10, 271-278.

9. Craft, L.L., Magyar, T.M., Becker, B.J. y Feltz, D.L. (2003). The rela- tionship between the competitive state anxiety inventory- 2 and sport performance: A meta-analysis. Journal of Sport and Exercise Psychology, 25, 44-65.

10. Cox, R.H., Martens, M.P. y Russell W.D. (2003). Measuring anxiety in athletics: The revised Competitive State Anxiety Inventory-2. Journal of Sport and Exercise Psychology, 25, 519-533.

11. De la Vega, R. (2010). Instrumentos aplicados en la evaluación psicológica realizada en el deporte. [Reseńa del libro: Roffé, M. (2009). Evaluación psicodeportológica. 30 Test psicométricos y proyectivos. Buenos Aires: Lugar Editorial].

12. Ehrlenspiel, F., Brand, R. y Graf, K. (2009). Das Wettkampf-AngstInventar-State. En R. Brand, F. Ehrlenspiel y K. Graf (Eds.), Das Wettkampf-Angst-Inventar. Manual (pp. 71-99). Bonn: BISp.

13. Endler, N.S. y Kocovski, N.L. (2001). State and trait anxiety revisted. Journal of Anxiety Disorders, 15, 231-241.

14. García-Mas, A., Sampol, P., Smith, R.E., Ponseti, J., Almeida, P., Lameiras, J., Jiménez, R. y Leiva, A. (2011). Ansiedad competitiva y clima motivacional en jóvenes futbolistas de competición, en relación con las habilidades y el rendimiento percibido por sus entrenadores. Revista de Psicología del Deporte, 20 (1), 197-207.

15. Grossbard, J.R., Smith, R.E., Smoll, F.L. y Cumming, S.P. (2009). Competitive anxiety in young athletes: Differentiating somatic anxiety, 
worry and concentration disruption. Anxiety, Stress and Coping, 22 (2), 153-166.

16. Guillén, F., Sánchez, R. y Márquez, R. (2003). La ansiedad en jugadoras espańolas de la Liga Espańola de Baloncesto. Archivos de Medicina del Deporte, XX (95), 213-220.

17. Guillén, F. y Sánchez, R. (2009). Competitive anxiety in expert female athletes: sources and intensity of anxiety in national team and first division Spanish basketball players. Perceptual and Motor Skills, 109 407-419.

18. Guillén, F., Santana, A. y Bara, M. (2006). Niveles de ansiedad en jugadores internacionales de baloncesto en silla de rueda. Revista Iberoamericana de Psicología del Ejercicio y el Deporte, 1 (1), 69-82.

19. Hackfort, D. y Schwenkmezger, P. (1989). Measuring anxiety in sport: Perspectives and problems. En D. Hackfort y C.D. Spielberger (Eds.), Anxiety in Sport: An International Perspective (pp. 55-74). NY: Hemisphere.

20. Hackfort, D. y Spielberger, C. D. (Eds.). (1989). Anxiety in Sports. An International Perspective. New York, NY: Hemisphere.

21. Hanton, S., Mellalieu, S.D. y Hall, R. (2002). Re-examining the competitive anxiety trait-state relationship. Personality and Individual Differences, 33, 1125-1136.

22. Hanton, S., Thomas, O. y Maynard, I. (2004). Competitive anxiety responses in the week leading up to competition: the role of intensity, direction and frequency dimensions. Psychology of Sport and Exercise, 5, 169-181.

23. Hoyle, R.H. (1995). Structural Equation Modeling. Concepts, Issues, and Applications. Thousand Oaks, California: Sage Publications.

24. Hu, L. y Bentler, P.M. (1999). Cutoff criteria for fit indexes in covariance structure analysis: Conventional criteria versus new alternatives. Structural Equation Modeling, 6, 1-55.

25. Jöreskog, K. G. y Sörbom, D. (2004). LISREL 8.71.: User's reference guide. Lincolnwood,

26. IL: Scientific Software.

27. Lévy-Mangin, J.P. (2006). Modelización con Estructuras de Covarianzas en Ciencias Sociales: Temas Esenciales, Avanzados y Aportaciones Especiales. Coruña: Netbiblo.

28. Martens, R., Vealey, R.S. y Burton, D. (1990). Competitive Anxiety in Sport. Champaign, IL: Human Kinetics.

29. MacCallum, R.C, Zhang, S., Preacher, K.J. y Rucker, D.D. (2002). On the practice of dichotomization of quantitative variables. Psychological Methods, 7, 19-40.

30. Mellalieu, S.D. y Hanton, S. (Eds.). (2009). A competitive anxiety review: Recent directions in sport psychology research. Hauppauge, NY: Nova Science.

31. Mellalieu, S.D., Neil, R., Hanton, S. y Fletcher, D. (2009). Competition stress in sport performers: Stressors experienced in the competition environment. Journal of Sports Sciences, 27, 729-744.

32. Neil, R., Mellalieu., S.D., Hanton, S. y Fletcher, D. (2011, en prensa). Competition stress and emotions in sport performers: The role of further appraisals. Psychology of Sport \& Exercise. doi:10.1016/j.psychsport.2011.02.001.

33. Olmedilla Zafra, A., Andreu Álvarez, M.D., Ortín Montero, F. J. y Blas Redondo, A. (2009). Ansiedad competitiva, percepción de éxito y lesiones: un estudio en futbolistas. Revista Internacional de Medicina y Ciencias de la Actividad Física y el Deporte, 9 (33), 51-66.

34. Partridge, J.A. y Wiggins, M.S. (2008). Coping styles for trait shame and anxiety intensity and direction in competitive athletes. Psychological Reports, 103, 703.

35. Patel, D.R., Omar, H. y Terry, M. (2010). Sport related Performance Anxiety in Young Female Athletes. Journal of Pediatric and Adolescent Gynecology, 23, 325-335.

36. Pons, D. (1994). Un estudio sobre la relación entre ansiedad y rendimiento en jugadores de golf. Tesis doctoral sin publicar. Universitat de Valencia.

37. Ramis, Y., Torregrosa, M., Viladrich, C. y Cruz, J. (2010). Adaptación y validación de la versión espańola de la Escala de Ansiedad Competitiva SAS-2 para deportistas de iniciación. Psicothema, 22 (4), 1004-1009.

38. Sarriugarte, F. (1999). Estudio de la relación entre el nivel de activación y el rendimiento deportivo en fútbol. En A. López de la Llave, M.C. Pérez y J.M. Busceta (Eds.), Investigaciones Breves en Psicología del Deporte (pp. 135-137). Madrid: Dykinson.

39. Schwenkmezger, P. (1985). Modelle der Eigenschafts und Zustandsangst. Göttingen, Germany: Hogrefe.

40. Smith, R.E., Smoll, F.L. y Schutz, R.W. (1990). Measurement and correlates of sport-specific cognitive and somatic trait anxiety: The sport anxiety scale. Anxiety Research, 2, 263-280.

41. Smith, R.E., Smoll, F.L., Cumming, S.P. y Grossbard, J.R. (2006) Measurement of multidimensional sport performance anxiety in children and adults: The sport anxiety scale-2. Journal of Sport and Exercise Psychology, 28, 479-501.

42. Smith, R.E., Schutz, R.W., Smoll, F.L. y Ptacek, J.T. (1995). Development and validation of a multidimensional measure of sport specific psychological skills: The Athletic Coping Skills Inventory-28. Journal of Sport and Exercise Psychology, 17, 379-398.

43. Spielberger, C.D. (1966). Theory and research on anxiety. En C.D. Spielberger (Ed.), Anxiety and Behavior (pp. 3-22). New York: Academic Press.

44. Spielberger, C.D. (1972). Anxiety as an emotional state. En C.D. Spie lberger (Ed.), Anxiety Behavior (pp. 23-49). New York: Academic Press.

45. Spielberger, C.D. (1989). Anxiety in sports: An international perspective. New York: Hemisphere Publishing Corporation.

46. Thomas, O., Picknell, G. y Hanton, S. (2011). Recall agreement between actual and retrospective reports of competitive anxiety: A comparison of intensity and frequency dimensions. Journal of Sports Sciences, 29, 495-508

47. Wadey, R. y Hanton, S. (2008). Basic psychological skills usage and competitive anxiety responses: perceived underlying mechanisms. Research Quarterly for Exercise and Sport, 79, 363-373.

48. Woodman, T. y Hardy, L. (2003). The relative impact of cognitive anxiety and self-confidence upon sport performance: A meta-analysis. Journal of Sports Sciences, 21, 443-457.

49. Ziegler, M., Ehrlenspiel, F. y Brand, R. (2009). Latent state-trait theory: An application in sport psychology. Psychology of Sport and Exercise, 10, 344-349. 